ARTÍCULO

\title{
Hacia la prevención del ausentismo escolar: propuestas para la intervención socioeducativa*
}

\author{
Alicia Razeto Pavez' (D)
}

\section{RESUMEN}

El ensayo tiene por objetivo visibilizar la problemática del ausentismo escolar en los centros educativos, definiéndolo no solo como la cantidad de días faltados a clase sino que como un complejo proceso de ruptura escolar, de profundas y variadas causas. También se analiza la relevancia que tiene la puesta en marcha de estrategias preventivas de intervención, tempranas y oportunas, de modo de evitar las consecuencias perjudiciales que puede traer el ausentismo en los niños y adolescentes. Finalmente, se proponen un conjunto de condiciones que las actuaciones preventivas de los centros educativos en torno al absentismo escolar pueden considerar en su formulación, con miras a favorecer la reducción de la presencia de este problema.

\section{PALABRAS CLAVE}

asistencia a la escuela; programas de prevención; intervención social.

\footnotetext{
*Este artículo se encuentra asociado al estudio Fondo Nacional de Desarrollo Científico y Tecnológico (FONDECYT) Regular n. 1200338, Agencia Nacional de Investigación y Desarrollo (ANID), Chile. La autora del artículo también agradece el apoyo de Postdoctorado Becas Chile 74180055, Comisión Nacional de Investigación Científica y Tecnológica (CONICYT), Chile. 'Pontificia Universidad Católica de Chile, Santiago, Chile.
} 


\section{TOWARD THE PREVENTION OF SCHOOL ABSENTEEISM: PROPOSALS FOR SOCIO-EDUCATIONAL INTERVENTION}

\section{ABSTRACT}

The aim of the article was to make visible the problem of school absenteeism in schools, defining it not only as the number of days missed from school, but also as a complex process of school rupture, with deep and varied causes. The relevance of the implementation of preventive intervention strategies, early and timely, in order to avoid the harmful consequences that absenteeism can bring to children and adolescents is also analyzed. Finally, a set of conditions are proposed that the preventive actions of schools regarding school absenteeism may consider in their formulation, with a view to favoring the reduction of the presence of this problem

KEYWORDS

attendance at school; prevention programs; social intervention.

\section{RUMO À PREVENÇÃO DO ABSENTISMO ESCOLAR: PROPOSTAS PARA A INTERVENÇÃO SOCIOEDUCATIVA}

RESUMO

O artigo teve por objetivo visibilizar a problemática do absentismo escolar nos centros educativos, definindo-o não só como a quantidade de dias perdidos na escola, mas como um complexo processo de ruptura escolar, de causas profundas e variadas. Também se analisa a relevância da implementação de estratégias preventivas de intervenção, precoces e oportunas, de modo a evitar as consequências nocivas que o absentismo pode trazer para crianças e adolescentes. Finalmente, propõe-se um conjunto de condições que as ações preventivas dos centros educativos em torno do absentismo escolar podem considerar em sua formulação, com vistas a favorecer a redução da presença desse problema.

PALAVRAS-CHAVE

assistência à escola; programas de prevenção; intervenção social. 


\section{INTRODUCCIÓN}

El ausentismo escolar se define habitualmente como la inasistencia reiterada o prolongada a clases durante el año escolar de un estudiante de primaria o secundaria matriculado en un centro educativo. Suele ser descrita como una variable de stock, medida en términos de días faltados, pero el ausentismo es un fenómeno mucho más complejo y profundo, que pone en evidencia un proceso de ruptura con la escuela por parte del estudiante y su familia (García, 2013). Este proceso de ruptura puede comenzar con la inasistencia esporádica a clases hasta profundizarse con la decisión de abandonar la escuela. La inasistencia es una muestra del desapego, desenganche o una desvinculación de los niños y jóvenes con la institución escolar, para quienes la educación representa algo secundario en sus vidas puesto que han visto mermado su valor tanto instrumental como expresivo (Fernández Enguita, 2011).

Si bien se ha avanzado en detectar las causas del ausentismo, menos se ha progresado en aportar programas de intervención o soluciones que evidencien su eficacia en resolver el problema (Reid, 2008). Esta escasez de actuaciones y evaluaciones de efectividad es más pronunciada en la región latinoamericana. En parte, esto puede obedecer a que el ausentismo escolar es "un proceso biográfico que esconde una gran variedad de situaciones personales, sociales y educativas" (García, 2013, p. 27). Es un problema complejo que se produce por la interacción de varios factores provenientes de distintos ámbitos, como los individuales, escolares, familiares y de la comunidad. Los pocos avances en la solución del ausentismo también obedecen a que, a nivel del centro educativo, suele afectar a una proporción reducida de estudiantes en comparación con el total, y lidiar con sus consecuencias es costoso y consume tiempo de los profesionales y autoridades locales (Reid, 2008).

La intervención preventiva desde la escuela, temprana y permanente, es central para disminuir el ausentismo escolar y contribuir a un mejor proceso de escolaridad para los niños y jóvenes, anticipándose a la manifestación del problema y las consecuencias perjudiciales que puede producir en el futuro desarrollo de los estudiantes. Pese a esto, es habitual que los centros educativos activen protocolos de actuación frente al ausentismo escolar cuando este ya se ha cronificado, es decir, cuando el estudiante ha faltado a clases de forma prolongada en el tiempo. Es importante intentar revincular al niño o joven en estas situaciones, pero intervenir cuando el ausentismo es crónico se considera tardío y con menos posibilidad de ser revertido a que si se interviniese de manera temprana y preventiva. Esto es debido a que ya se ha disminuido el hábito de asistir a la escuela, se ha debilitado el vínculo con los profesores y compañeros y se va perdiendo progresivamente el interés por el aprendizaje.

El presente ensayo tiene por objetivo proponer un conjunto de condiciones que las actuaciones preventivas de los centros educativos en torno al absentismo escolar pueden considerar en su formulación, con miras a favorecer la reducción de la presencia de este problema en los centros educativos. 


\section{AUSENTISMO COMO PROCESO DE RUPTURA CON LA ESCUELA}

Para aportar a un debate crítico y reflexivo sobre el ausentismo escolar y las intervenciones socioeducativas, es preciso alejarse de ciertas visiones comunes y predominantes, afines con la teoría del hándicap sociocultural (García, 2013) como las que responsabilizan únicamente a la estructura o dinámica de las familias de los estudiantes o a su pertenencia a un determinado estrato social. Aunque sean factores que influencian en el absentismo, restringir el análisis desde una perspectiva del déficit familiar, empobrece la mirada, agudiza los estigmas, reduciendo las posibilidades de pensar en actuaciones efectivas sobre el ausentismo, basadas en la confianza y los recursos comunes con las familias y la comunidad local.

La escuela también tiene una responsabilidad en la producción del ausentismo escolar. De hecho, Strand (2014, p. 262) lo define como una

reacción saludable a un ambiente escolar poco amigable o como una manifestación de la insatisfacción con el sistema escolar [...] también puede ser visto como el resultado de circunstancias de vida de alto riesgo con consecuencias directas tales como el aislamiento social, fracaso escolar, adicción a las drogas o incluso el crimen.

El ausentismo escolar puede considerarse como un proceso de ruptura escolar de naturaleza dinámica, temporal y multicausal. Según García (2009) este es un proceso dinámico, interactivo y heterogéneo, en sus perfiles y causas, capaz de manifestarse bajo múltiples formas e intensidades, como el retraso reiterado, el ausentismo esporádico, moderado, crónico, o incluso hasta el abandono temprano de la escuela.

El proceso de ruptura con la escuela es progresivo y se puede dar por diversos factores. Como señala García (2005) uno de los principales factores que produce el ausentismo se encuentra en el propio centro educativo, por las prácticas pedagógicas de los centros y las políticas socioeducativas (García, 2005). Hay varios estudios que demuestran que el proceso de desapego del estudiante con la escuela se origina por relaciones conflictivas con los profesores, compañeros de clase o el ambiente en general del centro educativo. Así por ejemplo, un estudio de Strand (2014) concluye que las principales razones que dan los jóvenes a su ausencia se relacionan a factores relacionados con el ambiente escolar, como la falta de relaciones sociales con sus pares y profesores, no entender las instrucciones del profesor, no tener amigos, tener conflictos en el colegio o aburrirse en clases. Un estudio de Reid (2008) afirma que hay estudios que sugieren que los absentistas "están aburridos con la escuela, a menudo se sienten insatisfechos, no les gustan algunas materias que ven poco relevante, y necesitan apoyo con sus deficiencias en lenguaje y matemáticas" (Reid, 2008, p. 347).

Otro estudio establece que hay factores relacionados a la escuela que influyen fuertemente en el absentismo, como lo es el bullying y el acoso escolar (Prakash et al., 2017). Este mismo aspecto, el ambiente escolar inseguro, también es indicado como un factor de riesgo de absentismo escolar por un estudio de Mallett (2016) quien 
agrega que un factor protector de la asistencia a clases es que la escuela cuente con un servicio de prevención de la inasistencia escolar, con estrategias de motivación para el alumnado de riesgo, y con un plan de alianza con agentes de la comunidad local (Mallett, 2016).

Asimismo, Esterle-Hedibel (2005, p. 4) identifica a las dificultades escolares como uno de los factores que acompañaban a los procesos de ruptura escolar, aún más, "cuando la interacción con los profesores les confirma su nulidad en términos de resultados escolares, el riesgo de desescolarización es presente". Por otra parte, la relación del estudiante con el profesor es determinante en el proceso de ruptura, puesto que la "desviación implica necesariamente dos actores y que el profesor puede provocar o atenuar la desviación, con el estilo de su relación con sus alumnos. Distingue los 'provocadores' de desviación de los 'aisladores' de desviación" (Esterle-Hedibel, 2005, p. 5).

Otros factores identificados por Mallett (2016) a nivel de la escuela y que influyen en el desarrollo del absentismo son el ambiente escolar negativo o inseguro, ubicación académica inapropiada, victimización por bullying, políticas de asistencias pobres, políticas disciplinas coercitivas. London, Sánchez y Castrechini (2016) añaden otros factores escolares como la falta de una relación positiva con el personal de la escuela y tener lazos con pares que han tenido conductas delictivas.

De acuerdo con García (2013) para evitar el desencadenamiento de problemas de desafección escolar, es fundamental que el profesor genere un clima acogedor en el aula el cual facilitará la integración de los estudiantes. También es importante favorecer la autoestima, haciendo que los estudiantes se sientan apoyados y valorados y evitando etiquetar conductas o estigmatizar. Reid (2008) va más allá y propone que la escuela necesitaría cambiar sus reglas, estructura y organización para evitar el absentismo.

\section{HACIA UNA MIRADA PLURICAUSAL DEL AUSENTISMO ESCOLAR}

Para formular actuaciones preventivas eficaces es central reconocer que habrá que actuar en otras esferas de la vida del estudiante, más allá de la educativa, en las que se encuentran factores fuertemente influyentes en la producción del absentismo. Se trata de la familia y el vecindario o la comunidad.

Según London, Sánchez y Castrechini (2016) entre los factores familiares relacionados al absentismo están el conflicto familiar, la percepción de cohesión familiar, disciplina inefectiva e inconsistente en el hogar y tener solo un padre. Una revisión realizada por Esterle-Hedibel (2005) indica que más que el tipo de familia, influye la calidad de las relaciones entre sus miembros, los conflictos, las rupturas biográficas, las condiciones de vida y estigmatización pueden ser influyentes en los procesos de desescolarización. Mallett (2016) identifica como factores de riesgo familiares los problemas financieros o la pobreza la falta de transporte, no tener hogar, conflictos familiares significativos, maltrato, bajo apoyo parental y actitud de poca preocupación por la escuela. 
Por otra parte, las creencias de las familias sobre el absentismo del estudiante puede ser un factor que contribuye a este fenómeno. Rogers y Feller (2018) encontraron que las familias de estudiantes con un alto absentismo presentaban dos creencias erradas. Por una parte, subestiman la cantidad de ausencias totales, creyendo que son la mitad de las que realmente son. Por otra parte, no son conscientes de las ausencias relativas o comparadas con los compañeros de clase del estudiante.

Por su parte, London, Sánchez y Castrechini (2016) coinciden en los factores de la relación con los pares, el uso de drogas y el bajo rendimiento académico. También añaden como factores de riesgo la autoestima del estudiante, el autoconcepto académico, la baja aspiración educacional y el tener mucho tiempo no supervisado por un adulto después de la escuela.

El ausentismo también es influido por la comunidad del estudiante, tanto a nivel local (barrio) como en lo que respecta a la comunidad cultural. Por ejemplo, Mallett (2016) detecta que la ocurrencia de violencia cerca del entorno del hogar y/o de la escuela, puede desencadenar absentismo. Por otra parte, las actitudes hacia la escuela están mediadas por las culturas de origen, pudiendo producir diferencias relevantes.

Río y Benítez (2009) identificaron en los discursos de algunos profesionales ciertas características familiares que aumentan las posibilidades de constituirse en hogares con niños absentistas. Estas fueron pertenecer al grupo étnico gitano, que mayoritariamente se dedica a la venta ambulante y en el que las niñas asumen tareas de cuidado doméstico y de cuidado de hermanos en reemplazo de sus madres, con el consecuente alejamiento de la escuela. También está el hecho de tener hermanos mayores absentistas, lo que supondría un mayor riesgo de reproducir la senda rupturista de los primogénitos.

En el ámbito de la comunidad, se ha encontrado que estudiantes que tienen redes de apoyo, recursos institucionales y participan de programas comunitarios (de servicio comunitario, enriquecimiento académico o aprendizaje emocional) fuera del horario escolar, tienen mejores tasas de graduación en secundaria (Dussaillant, 2017).

\section{LA PREVENCIÓN EN LAS INTERVENCIONES SOCIOEDUCATIVAS EN AUSENTISMO ESCOLAR}

El principal argumento para favorecer el desarrollo de actuaciones tempranas y preventivas sobre el ausentismo escolar es que tiene posibles consecuencias perjudiciales y que estas afectan a una población prioritaria como lo es la infancia y la adolescencia, menoscabando su proceso de escolarización y el derecho a la educación.

Se han realizado varios estudios que muestran el impacto que tiene el absentismo en el desempeño educativo y en el futuro proceso de integración social del estudiante. La mayoría de los investigadores concuerdan en el daño que produce porque, por un lado, afecta los resultados académicos de los estudiantes, y por otro, 
afecta a los profesores porque se produce un déficit de aprendizaje que les frustra o desmoraliza (Reid, 2008).

Con respecto a los resultados académicos de los estudiantes, estudios muestran que el absentismo quiebra la continuidad del aprendizaje, merma la autoconfianza del estudiante (Malcolm et al., 2003). Produce menos resultados en aprendizaje de matemáticas y lectura, menos motivación con los estudios y menor adquisición de habilidades sociales, propias del desarrollo de un grupo social, impactando en mayor dificultad para hacer y mantener amistades (Gottfried, 2014; Malcolm et al., 2003).

Una publicación de Balfanz y Byrnes (2012) reúne los resultados de un conjunto de estudios realizados en seis estados de Estados Unidos sobre el impacto del absentismo crónico, concluyendo que tiene efectos negativos en todos los niveles de enseñanza (preprimaria, primaria y secundaria) y que se resumen en que el absentismo crónico:

- Disminuye el desempeño de los estudiantes, acentuándose en los que provienen de familias de bajos ingresos, disminuyendo las habilidades en matemáticas, lectura y escritura.

- Cuando se da en prekínder o kínder, aumenta la posibilidad que este continúe durante la enseñanza primaria.

- Cuando se da en secundaria, además de afectar negativamente los resultados en pruebas en matemáticas y lectura, interrumpe y afecta las expectativas de los tiempos de graduación y aumenta las probabilidades del abandono educativo.

Por otra parte, un estudio realizado en California, Estados Unidos, por London, Sánchez y Castrechini (2016) encontró que había una brecha continua a través de los años en los resultados de una prueba estandarizada de aprendizaje, en matemática y lenguaje, atribuible al absentismo crónico.

Pero los efectos del absentismo no solo se reducen a los estudiantes, sino que también trastocan a los profesores y a los compañeros de clase. Los profesores ven desviada su atención del resto de la clase y debe brindarle de su tiempo libre para ayudar al absentista a integrarse al aprendizaje (Malcolm et al., 2003). La situación de absentismo reporta incertidumbre, el profesor habitualmente no conoce la razón de la ausencia ni su momento de reincorporación. Una vez que retorna, el profesor puede sentirse frustrado o desmoralizado (Malcolm et al., 2003) por no saber cómo apoyarlo o no poder hacerlo como quisiera de modo tal de no perjudicar el avance de él ni del grupo.

Con respecto a los compañeros de clase, estudios realizados por Gottfried $(2011,2015)$ muestran que el menor grado de desempeño en las pruebas estandarizadas también incluye a los compañeros de clase de los estudiantes absentistas. Esto también lo confirma un estudio la Organisation for Economic Co-operation and Development (OECD, 2016) a partir de los resultados Programme for International Student Assessment (PISA) de 2015, el que señala que el desempeño en ciencia es peor no solo por parte de los absentistas, sino que de toda la clase. Durante el tiempo de ausencia, los compañeros de clase han perdido la oportunidad 
de interactuar con los estudiantes absentistas. Además, estos se ven afectados por la desviación de tiempo y disrupción de la clase cuando el alumno absentista retorna (Malcolm et al., 2003).

El ausentismo también impacta el proceso de integración social del niño o adolescente, porque no solo afecta las posibilidades de progresión escolar y culminación exitosa del ciclo educativo, sino que también las posibilidades futuras de empleo, promoción personal y profesional, pudiendo acrecentar las probabilidades de marginación, paro, delincuencia, etc. (González González, 2005). Asimismo, este impacto no solo se reducirá al estudiante absentista sino que también a su núcleo familiar, pudiendo llegar a mermar el desarrollo futuro de sus miembros.

Todas estas evidencias ponen de manifiesto la relevancia que tiene desarrollar estrategias de prevención efectivas en contra de este fenómeno. El eje central de los programas de intervención consiste en anticiparse a la aparición de problema, interviniendo en situaciones de riesgo, o abordarlo tan pronto como comienzan a manifestarse sus primeros indicios.

Existen innumerables situaciones de riesgo psicosocial que hacen más probable que un estudiante se ausente o definitivamente abandone la escuela. Entre estas, el embarazo y la maternidad o paternidad temprana, la drogodependencia, la violencia escolar, depresión o problemas de salud mental, entre otros. A nivel familiar, también se encuentran las situaciones de inmigración, en los que irrumpen los procesos de reencuentro familiar y duelo migratorio, con el impacto emocional, y los viajes al país de origen durante el curso escolar, con la interrupción del proceso de escolaridad. A nivel escolar, la acumulación de las amonestaciones o expulsiones del centro escolar también son situaciones de riesgo, por el debilitamiento del vínculo con la escuela y el impacto en el autoestima del estudiante.

La detección oportuna de estas situaciones personales, familiares y escolares por parte del centro educativo es central para poder desarrollar actuaciones de estrategias preventivas. También es probable que no sea el centro educativo el primer o único agente en detectar estas situaciones, con lo que es fundamental poseer una comunicación permanente y trabajo en red con los centros de salud $u$ otros organismos pertinentes para estar enterado de estas situaciones y activar actuaciones de acompañamiento de la escuela para apoyar el proceso de escolaridad de estos estudiantes, evitando situaciones de ausentismo o desenlaces de abandono.

\section{CONDICIONES DE LAS ESTRATEGIAS PARA LA PREVENCIÓN DEL AUSENTISMO ESCOLAR}

Si bien han proliferado experiencias de programas de intervención socioeducativos en ausentismo escolar, hay pocas evaluaciones de la efectividad de estos (Cook et al., 2017). No obstante, es posible proponer algunas condiciones claves de los programas de prevención de absentismo. La posibilidad de asumir 
estas condiciones en las estrategias preventivas dependerá de las características y dinámicas de los centros educativos, tales como su estructura, organización interna, recursos, cultura y clima escolar. La flexibilidad es, ante todo, un principio de base en la formulación y aplicación de estas actuaciones.

\section{COORDINACIÓN Y TRABAJO EN RED}

Es improbable que la sola actuación del centro educativo alcance resultados efectivos y sostenibles en un problema de causas tan variadas y profundas como es el ausentismo escolar. Por eso es que se requiere de un trabajo en red y coordinado de los centros educativos con otros agentes socioeducativos del territorio, como lo es el municipio, el cual dispone de programas y recursos variados dirigidos al bienestar de la población de niños y jóvenes de la comunidad local. También es el caso de los centros de salud y la policía local. Al mismo tiempo, se puede involucrar activamente a organizaciones no gubernamentales que tengan una actuación en el territorio y un reconocido interés en el tema educativo.

Lograr la articulación de actuaciones de agentes distintos supone la necesidad de compartir sistemáticamente información, establecer y definir los compromisos y las responsabilidades, entre las cuales está el liderazgo de este trabajo colectivo, que se espera pueda asumirlo el centro educativo. La noción de corresponsabilidad en la prevención del absentismo escolar, es uno de los principios rectores para la implementación de este tipo de programas según García (2013). De acuerdo con esta autora:

la corresponsabilidad es un principio y criterio de actuación que presupone un compromiso compartido entre diferentes administraciones para el cumplimiento de los deberes y obligaciones relativos a la garantía del derecho a la educación. Se fundamenta en el compromiso, cooperación y participación desde la proximidad, de los diferentes agentes educativos. (García, 2013, p. 128)

La noción de corresponsabilidad se puede materializar mediante la metodología del trabajo en red, a través de la cual diversos agentes socioeducativos del territorio se comunican y reúnen periódicamente, con el fin de planificar, aplicar, hacer seguimiento y evaluación las actuaciones en torno al ausentismo escolar. Un elemento central para afrontar un trabajo en red, en torno a un problema socioeducativo complejo y con un enfoque preventivo, es el trabajo en equipo e interdisciplinario, integrado por profesionales y técnicos del área educativa, psicológica, social y legal. Entre los resultados que pueden obtenerse a partir de un trabajo de esta naturaleza está la creación de planes municipales para combatir el ausentismo, los que son según García (2013, p. 101):

un dispositivo local de intervención, es decir, un mecanismo articulado en el territorio diseñado para combatir el absentismo escolar mediante actuaciones de 
prevención, detección, reparación y seguimiento de situaciones de absentismo así como la evaluación de la eficacia de las intervenciones desarrolladas por la mejora del dispositivo.

\section{FAMILIAS Y COMUNIDAD LOCAL COMO ALIADOS EN LA PREVENCIÓN DEL AUSENTISMO ESCOLAR}

Como se revisó en secciones anteriores, el ausentismo se origina, en parte, por situaciones que se producen en el seno de la familia. También contribuyen a este elementos y dinámicas propias de la comunidad local. Pero esta no es razón para criticarlos ni excluirlos del trabajo para erradicar el ausentismo, sino que todo lo contrario, es central visualizarlos como potenciales aliados en el éxito del proceso educativo de los niños y adolescentes, tal como lo propone numerosa literatura en la materia de relación escuela-familia-comunidad local. Estudios sobre eficacia escolar han comprobado el valor que tiene la relación de las familias con las escuelas (Murillo, 2008). Deal y Peterson (2009) concluyen que uno de los hallazgos más consistentes es que la participación de los padres provoca una diferencia significativa en el desempeño educativo de los estudiantes y también en la gestión del staff del establecimiento. En la misma línea, Harris (2009) establece que el involucramiento de los padres como aliados de las escuelas es una de las estrategias fundamentales de los modelos de mejoramiento escolar en establecimientos educativos vulnerables. Por esta razón es que es de central importancia involucrar activamente a estos dos agentes en los programas de prevención del ausentismo escolar, a través de la información, la sensibilización, el trabajo educativo y la cooperación en la disminución de este fenómeno.

Existen diversos modelos y estrategias de participación de las familias en la educación de sus hijos (Epstein, 2011; Weiss et al., 2009) posibles de aplicar en los centros educativos. Investigaciones actuales en el campo educacional proponen a la visita domiciliaria, como una estrategia innovadora y efectiva para tomar contacto y establecer un vínculo con familias más distantes del centro educativo (Razeto, 2016) y que supone un encuentro con las familias fuera de los espacios tradicionales intraescuela.

Desde el enfoque preventivo, las visitas domiciliarias pueden tener por objetivo, por un lado, tomar contacto con las familias distantes de niños absentistas y conocer las causas del absentismo, y por otro lado, realizar un trabajo educativo con las familias que permitan reforzar prácticas que fomenten el interés y compromiso de asistencia a la escuela. Una de las principales fortalezas de esta herramienta, es el desarrollo de un vínculo entre el agente visitador y la madre o padre, razón por la cual, es importante no sea aplicada como una medida aislada o excepcional sino que se mantenga en el tiempo si es que se considera necesario, o que se complemente con la aplicación de otras herramientas de trabajo (Razeto, 2018). 
Por otra parte, está la comunidad local como aliado de la tarea educativa de la escuela (Krichesky, 2006). En la prevención del absentismo los agentes del barrio pueden tener una función muy importante, portando un discurso que valore y promueva la asistencia a la escuela de los niños de la comunidad local y ofertando oportunidades para la participación y desarrollo integral de niños y jóvenes. Entre estos agentes están los comerciantes, los trabajadores municipales, los vecinos del barrio y las organizaciones sociales y comunitarias. Entre los espacios más significativos de la comunidad se encuentran las plazas y parques, centros comunitarios, bibliotecas locales, entre otros.

\section{TRANSICIONES EDUCATIVAS COMO MOMENTO DE PREVENCIÓN}

La relevancia y complejidad que tiene el proceso de transición entre la educación primaria y secundaria ha sido estudiado por muchos investigadores. Monarca, Rappoport y Fernández (2012) describen que para un grupo de jóvenes la transición puede no ser un problema mientras que para otro puede representar un gran problema, que puede generar consecuencias con efectos impredecibles en las trayectorias educativas, como activar temporadas de ausentismo o desenlaces de abandono escolar temprano. La transición es un proceso de cambios y acomodaciones, un proceso con distintos signos, ambivalente (Gimeno, 1997; Monarca, Rappoport y Fernández González, 2012). Para los jóvenes con mayor vulnerabilidad, la transición exige una respuesta educativa, la implementación de medidas tendientes a facilitar la transición (Monarca y Gallego, 2010; Monarca, Rappoport y Fernández González, 2012). Según Ross (2016) lamentablemente hay pocas prácticas para apoyar a los jóvenes en la transición de primaria a secundaria y escasa información de programas para incrementar el apoyo de los padres en el período de transición.

Desde el enfoque preventivo del ausentismo escolar, es necesario desarrollar programas de acompañamiento psicosocial a los estudiantes y sus familias más vulnerables durante el período de transición, desarrollando espacios de conversación, orientación y apoyo socioeducativo. Es preciso considerarlo como momento de cambios importantes, en los cuales es clave prevenir situaciones de desescolarización y acompañar proceso de adaptación y vinculación a la secundaria. Un buen proceso de coordinación administrativo y pedagógico es necesario para asegurar la continuidad entre ambas etapas. Pero la transición no solo se reduce al paso de la primaria a secundaria, sino que también tiene otros momentos centrales, como los siguientes:

- El ingreso del niño al sistema de educación parvularia. Es un momento clave para acompañar a las familias y trabajar el valor de la escuela y la adhesión educativa.

- El paso de la educación parvularia a la primaria o básica. Con el objetivo de prevenir dificultades de rendimiento escolar y situaciones de absentismo durante la enseñanza primaria. 


\section{ACOGIDA Y REINCORPORACIÓN DEL ESTUDIANTE ABSENTISTA: MOMENTO CENTRAL DE PREVENCIÓN}

La reincorporación de un estudiante absentista es una importante oportunidad que tiene la escuela para conectarlo con el ambiente escolar y motivarlo con el aprendizaje. La vuelta del estudiante, su presencia en clases, es el resultado de un proceso que ha significado un esfuerzo para el estudiante y su familia, que la escuela debe reconocer, valorar y utilizar a favor de su reenganche. No obstante, la asistencia del estudiante no asegura ni garantiza su futura presencia en la escuela. Para que sea exitoso y sostenible se requiere un proceso de acompañamiento al estudiante y su familia por parte de la escuela, desde el primer día de retorno hasta lograr la normalización de su proceso escolar. Este proceso de acogida y acompañamiento involucra y compromete al tutor, profesores, jefe pedagógico y equipo psicosocial de la escuela, quienes en conjunto con el estudiante y su familia, pueden formular un Plan de Reincorporación y Acogida para el estudiante absentista.

Este plan tiene el objetivo de favorecer la mejor reintegración del estudiante absentista a la escuela, promoviendo la comunicación, organización y flexibilidad en este proceso. Con relación a la comunicación, el plan tiene por objetivo evitar que el profesor del estudiante y su grupo de compañeros se vean sorprendidos por la reincorporación del estudiante, comunicándoles su fecha estimada de reincorporación, sugiriéndoles minimizar la expresión de frases o preguntas que pudiesen incomodar al estudiante que se reincorpora y recomendando los gestos de acogida por parte de estos. Con respecto a la organización, el plan permite que el profesor planifique las actividades pedagógicas prioritarias para lograr paulatinamente la nivelación del estudiante con respecto al grupo de clase. Con relación a la flexibilidad, el plan permite establecer tiempos y exigencias paulatinas y relativas al grado de absentismo que el estudiante haya presentado. De esta manera se aminoran las posibilidades que el estudiante se sienta sobre exigido.

\section{CONCLUSIONES}

$\mathrm{El}$ ausentismo escolar es un problema socioeducativo que se produce por la conjunción de un grupo de factores escolares, familiares y del vecindario que pueden marcar significativamente el presente y las posibilidades futuras de desarrollo social y educativo de los niños y adolescentes. Desde la perspectiva de la ciudadanía y de derechos sociales, el absentismo imposibilita el cumplimiento del derecho a la educación. Asimismo, el absentismo pone en jaque a todo el sistema de educación público, puesto que este se basa en el principio que los estudiantes asisten regularmente, día a día, a la escuela. Sin la presencia del alumnado en el aula, no es posible el aprendizaje tal y como ha sido concebido por la escuela tradicional, en la cual existe un profesor que enseña, a lo largo del año escolar y a través de diversas actividades guiadas por un currículum, a un grupo de estudiantes. 
Un absentismo exacerbado, significaría el sinsentido y fracaso de la escuela y del proceso educativo.

Es fundamental avanzar en la formulación e implementación de experiencias de prevención del ausentismo en las escuelas. La prevención implica una intervención temprana y oportuna, permanente y a lo largo de todo el ciclo escolar, que permita detectar situaciones de riesgo y contribuir al enganche del alumno a la escuela. Este ensayo ha propuesto cuatro condiciones centrales que las actuaciones preventivas en ausentismo podrían incorporar para su mayor efectividad: la coordinación y el trabajo en red; contar con la familia y la comunidad local como aliados en la prevención del ausentismo y; considerar como momentos claves de la prevención el proceso de transición educativa y la reincorporación del alumno absentista a la sala de clases.

Además de todos estos elementos, es fundamental que la escuela revise y mejore las dinámicas pedagógicas y relaciones interpersonales en el aula de clase, que son una de las principales fuentes de satisfacción y enganche del estudiante con la escuela.

\section{REFERENCIAS}

BALFANZ, R.; BYRNES, V. Chronic absenteeism: summarizing what we know from nationally available data. Baltimore: Johns Hopkins University Center for Social Organization of Schools, 2012.

COOK, P.; DODGE, K.; GIFFORD, E.; SCHULTING, A. A new program to prevent primary school absenteeism: results of a pilot study in five schools. Children and Youth Services Review, United States, v. 82, p. 262-270, 2017. https://doi.org/10.1016/j. childyouth.2017.09.017

DEAL, T.; PETERSON, K. Shaping school culture: pitfalls, paradoxes, \& promises. San Francisco: Josey-Bass, 2009.

DUSSAILLANT, F. Deserción escolar en Chile. Propuestas para la investigación y la política pública. Análisis - Centro de Políticas Públicas, Chile, documento número 18, p. 1-18, jun. 2017. Disponible en: http://gobierno.udd.cl/cpp/files/2017/08/18Deserción.pdf. Acceso en: 12 dic. 2018.

EPSTEIN, J. School, family and community partnerships. United States: Westview Press, 2011.

ESTERLE-HEDIBEL, M. Prevención y tratamiento del absentismo y de la desescolarización en Francia: experiencias y nuevas formas de actuar. Revista Iberoamericana sobre Calidad, Eficacia y Cambio en Educación, v. 3, n. 1, p. 895905, 2005. Disponible en: http://www.redalyc.org/articulo.oa?id=55130182. Acceso en: 16 sept. 2018.

FERNÁNDEZ ENGUITA, M. Del desapego al desenganche y de este al fracaso escolar. Cadernos de Pesquisa, São Paulo, v. 41, n. 144, p. 732-751, 2011. Disponible en: http://www.scielo.br/pdf/cp/v41n144/v41n144a05.pdf. Acceso en: 22 mar. 2019. https://doi.org/10.1590/S0100-15742011000300005 
GARCÍA, M. Culturas de enseñanza y absentismo escolar en la enseñanza secundaria obligatoria: estudio de casos en la ciudad de Barcelona. Revista de Educación, Barcelona, n. 338, p. 347-374, 2005.

GARCÍA, M. El absentismo escolar: algunas claves para el desarrollo de intervenciones integradas en el marco de una escuela inclusiva y del territorio. In: BOLÍVAR, A. Absentismo y abandono escolar: políticas y buenas prácticas. Madrid: Book Chapter, 2009. p. 4-5. (Monográficos Escuela). Disponible en: https://www.researchgate.net/ profile/Antonio_Bolivar/publication/286884137_Absentismo_y_abandono_escolar_ Politicas_y_buenas_practicas/links/566ee8c108ae4d4dc8f7d999/Absentismo-yabandono-escolar-Politicas-y-buenas-practicas.pdf. Acceso en: 20 jun. 2020.

GARCÍA, M. Absentismo y abandono escolar. Madrid: Editorial Síntesis, 2013.

GIMENO, J. La transición a la educación secundaria. Discontinuidades en las culturas escolares. Madrid: Editorial Morata, 1997.

GONZÁLEZ GONZÁLEZ, M. T. El absentismo y el abandono: una forma de exclusión escolar. Profesorado - Revista de Currículum y Formación del Profesorado, España, v. 9, n. 1, p. 1-12, 2005. Disponible en: https://recyt.fecyt.es/index.php/ profesorado/article/view/42396/24325. Acceso en: 20 jun. 2020.

GOTTFRIED, M. A. Absent peers in elementary years: the negative classroom effects of unexcused absences on standardized testing outcomes. Teachers College Record, Pennsylvania, v. 113, n. 8, 1.597-1.632, 2011.

GOTTFRIED, M. A. Chronic absenteeism and its effects on students' academic and socioemotional outcomes. Journal of Education for Students Placed at Risk, United Kingdom,v. 19, n. 2, p. 53-75, 2014. http://dx.doi.org/10.1080/10824669.2014.962696 GOTTFRIED, M. A. Chronic absenteeism in the classroom context: effects on achievement. Urban Education, United States, v. 54, n. 1, p. 3-34, 2015. http://dx.doi. org/10.1177/0042085915618709

HARRIS, A. Improving schools in challenging contexts. Second International Handbook of Educational Change. Netherlands: Springer, 2009. p. 693-706.

KRICHESKY, M. Escuela y comunidad: desafíos para la inclusión educativa. 1. ed. Buenos Aires: Ministerio de Educación, Ciencia y Tecnología de la Nación, 2006.

LONDON, R.; SÁNCHEZ, M.; CASTRECHINI, S. The dynamics of chronic absence and student achievement. Education Policy Analysis Archives, United States, v. 24, n. 112, p. 1-27, 2016. https://doi.org/10.14507/epaa.24.2471

MALCOLM, H.; WILSON, V.; DAVIDSON, J.; KIRK, S. Absence from school: a study of its causes and effects in seven LEAs. United Kingdom: The SCRE Centre, University of Glasgow, 2003. (Research Report, 424). Disponible en: http://dera.ioe. ac.uk/8655/1/RR424.pdf. Acceso en: 2 mar. 2019.

MALLETT, C. Truancy: it's not about skipping school. Child and Adolescent Social Work Journal, United States, v. 33, p. 337-347, 2016. https://doi.org/10.1007/s10560015-0433-1

MONARCA, H. A.; GALLEGO, J. R. Tránsito a la ESO, ¿continuidad o ruptura?. Cuadernos de Pedagogía, Barcelona, n. 401, p. 28-31, mayo 2010. Disponible en: 
https://iesrdguezmonino.educarex.es/recursos/orientacion/TRANSITOP.pdf. Acceso en: 13 enero 2019.

MONARCA, H. A.; RAPPOPORT, S.; FERNÁNDEZ GONZÁLEZ, A. Factores condicionantes de las trayectorias escolares en la transición entre enseñanza primaria y secundaria. REOP, Madrid, v. 23, n. 3, p. 49-62, 2012. Disponible en: https:// repositorio.uam.es/bitstream/handle/10486/662035/factores_monarca_REOP_2012. pdf?sequence=1. Acceso en: 20 nov. 2018. https://doi.org/10.5944/reop.vol.23. num.3.2012.11461

MURILLO,J. Enfoque, situación y desafíos de la investigación sobre eficacia escolar en América. Eficacia escolar y factores asociados en América Latina y el Caribe. Santiago de Chile: UNESCO, 2008.

OECD - Organisation for Economic Co-operation and Development. PISA 2015. Pisa Resultados Clave. España: OECD, 2016. Disponible en: https:/www.oecd.org/ pisa/pisa-2015-results-in-focus-ESP.pdf. Acceso en: 24 jun. 2020.

PRAKASH, R.; BEATTIE, T.; JAVALKAR, P.; BHATTACHARJEE, P.; RAMANAIK, S.; THALINJA, R. et al. Correlates of school dropout and absenteeism among adolescent girls from marginalized community in north Karnataka, south India. Journal of Adolescence, United Kingdom, v. 61, p. 64-76, 2017. https://doi. org/10.1016/j.adolescence.2017.09.007

RAZETO, A. Estrategias para promover la participación de los padres en la educación de sus hijos: el potencial de la visita domiciliaria. Estudios Pedagógicos, Valdivia, v. 42, n. 2, p. 449-462, 2016. http://dx.doi.org/10.4067/S0718-07052016000200026

RAZETO, A. De trabajadores sociales y visitas domiciliarias en el sistema escolar chileno. Revista Katálysis, Florianópolis, v. 21, n. 2, p. 396-405, 2018. http://dx.doi. org/10.1590/1982-02592018v21n2p396

REID, K. The search for solutions to truancy and other forms of school absenteeism. Pastoral Care in Education, United Kingdom, v. 21, n. 1, p. 3-9, 2008. https://doi. org/10.1111/1468-0122.00248

RÍO RUIZ, M.; BENÍTEZ, J. Intervención socioeducativa y configuraciones familiares alejadas de la norma escolar: un estudio sobre absentismo y otras desimplicaciones parentales en la escolaridad. Sevilla: Centro de Estudios Andaluces, 2009.

ROGERS, T.; FELLER, A. Reducing student absences at scale by targeting parents' misbeliefs. Nature Human Behaviour, London, v. 2, p. 335-342, 2018. https://doi. org/10.1038/s41562-018-0328-1

ROSS, T. R. The differential effects of parental involvement on high school completion and postsecondary attendance. Education Policy Analysis Archives, United States, v. 24, n. 30, p. 1-38, 2016. https://doi.org/10.14507/epaa.24.2030

STRAND, A. "School — no thanks — it ain't my thing": accounts for truancy. Students' perspectives on their truancy and school lives. International Journal of Adolescence and Youth, United Kingdom, v. 19, n. 2, p. 262-277, 2014. https://doi.org/10.1080/0 2673843.2012.743920 
WEISS, H. B.; BOUFFARD, S. M.; BRIDGLALL, B. L.; GORDON, E. W. Reframing family involvement in education: supporting families to support educational equity. New York: Columbia University, 2009. (Equity Matters: Research Review n. 5). Disponible en: https://files.eric.ed.gov/fulltext/ED523994.pdf. Acceso en: 20 jun. 2020.

\section{SOBRE LA AUTORA}

Alicia Razeto Pavez es doctora en ciencias de la educación por la Pontificia Universidad Católica de Chile (Chile). Profesora de la misma institución.

E-mail: alicia.razeto@gmail.com

Recibido el 24 de agosto de 2019 Aprobado el 6 de abril de 2020 